\title{
Science on the up, down under
}

\author{
A series of national initiatives over the past 10 years has created a thriving nanoscience research \\ environment in Australia.
}

Australia is, to state the obvious, a long way from most academic hubs, such as those in Europe, the US, and, to a lesser extent, China. These vast geographical distances can mean that science in Australia receives limited attention abroad; it can also limit the migration of students and postdocs to and from the country. However, those that do venture there will find a nation that is investing heavily in science.

In nanoscience and technology the prospects for researchers are particularly rosy. A concerted effort in recent years by the federal government has led to several key initiatives in support of science at the nanoscale. The Australian Nanotechnology Network (ANN), for example, marks its tenth anniversary this year. This network, which is coordinated by Chennupati Jagadish of the Australian National University, is comprised of more than 400 research groups and aims to help foster collaborations between research groups in Australia and elsewhere, and to strengthen academic links to industry. The network promotes the sharing of national facilities, and supports the training of earlycareer scientists by funding short visits to host institutions and trips to conferences.

Another example of these initiatives is the Australian National Fabrication Facility (ANFF), which was established in 2007 with the aim of helping scientists in academia and industry to use and share state-of-the-art facilities. The facilities (which have more than 500 instruments) are located in 21 institutions across the country and provide all that is needed for fabrication and characterization in nanoelectronics, nanophotonics, advanced materials and nanobio applications. These facilities are accessible by users from any of the 21 institutions via an online booking system, and users can benefit from training and support from on-site technical staff.

Australia has a relatively small population of 23 million people. As a result, its education and research system relies on an influx of students and scientists from abroad to ensure that a critical mass is reached. And it is not always easy to attract the best candidates in a competitive global market. However, the size of the scientific community in Australia also means that collaborative efforts among institutions are frequently sought, and these collaborations can often be very effective, such as in the case of the ANN and the ANFF. In comparison, research efforts in places with a more competitive research culture, such as in Europe, can sometimes lead to unnecessary duplication of instrumentation and projects.

Federal government funding for science in Australia is principally distributed through the Australian Research Council (ARC) and the Commonwealth Scientific and Industrial Research Organisation (CSIRO), which is Australia's national science agency. In addition to federal government funding, state funding is also available, and some states are more supportive of the scientific ecosystem than others. The ARC has a scheme of fellowships to fund research projects by attracting world-renown researchers (Laureate Fellowships, 17 last year) and mid-career academics (Future Fellowships, 201 last year), and by supporting promising early-career academics (Discovery Early Career Researcher Award, 200 in 2014). In 2013, a total of AU\$522 million was allocated to fund 1,177 research projects through these and other schemes.

The ARC also co-funds several Centres of Excellence, which aim to be world-leading institutions in their respective fields and are funded for cycles of up to 7 years. Of particular relevance to nanotechnology is the ARC Centre of Excellence for Quantum Computation \& Communication Technology, which is directed by Michelle Simmons of the University of New South Wales. The centre was set up in 2011 and has since produced a number of notable results. Researchers at the centre have, for example, created a single-atom transistor by deterministically implanting a single phosphorus dopant atom in a silicon device (M. Fuechsle et al. Nature Nanotech. 7, 242-246; 2012), and manipulated a single nuclear spin in silicon as a quantum bit, with high fidelity in the read out of its state (J. J. Pla et al. Nature 496, 334-338; 2013). The ARC will also begin funding 12 new centres in 2014, with a total investment of AU\$285 million. One of those centres, the ARC Centre of Excellence for Nanoscale BioPhotonics, which will be directed by Tanya Monro of the University of Adelaide, will be dedicated to nanoscience and, in particular, to advanced light-based measurements in biological environments.

Despite these encouraging developments, there are some points of concern. The newly elected government has recently restructured departments related to higher education and research, with the CSIRO now falling under the remit of the Department of Industry, and the ANFF under the Department of Education. What this will mean for the availability of funding is still unclear, but some researchers fear it could lead to cuts in the funding of the national initiatives.

Another area of potential worry is the commercialization of academic research. Regardless of any political push towards economic growth, many researchers in the country consider the scientific culture to be risk averse, and the transfer of technology from academia to industry is certainly an area that could be improved. The typical commercialization path of university research in the country is licensing to existing companies, and it is rare to see Australian researchers creating start-ups. In this regard, Australia's geographic separation from more risk-taking approaches in the US and Europe also does not play to its advantage. The effects of any aversion to risk-taking are reinforced by the relatively small venture capital ecosystem and national market, which make it difficult to fund new enterprises. Furthermore, there are few industrial research and development centres in the country.

Strengthening the links with industry is seen as a priority in Australia. However, if the country wants to succeed in strengthening the role of research in the national economy, it would perhaps be wise to take some additional steps to support those researchers that want to try to commercialize their findings beyond a licensing model. Nevertheless, although the time may not yet be right for a commercialization thrust, there are at present numerous opportunities in the country for researchers in nanoscience and nanotechnology. And Australia's universities are on the lookout for the brightest international students and postdocs. 